Проблеми математичного моделювання та теорії диференціальних рівнянь

УДК 517.977.56

\title{
ЗАДАЧА ОПТИМАЛЬНОГО КЕРУВАННЯ ЕЛІПТИЧНОЮ СИСТЕМОЮ НА КЛАСІ НЕОБМЕЖЕНИХ МІР РАДОНА
}

\author{
С. О. Горбонос
}

Дніпропетровсъкий національний університет, кафедра диференціальних рівнянь, вул. Козакова, 18/14, ДНУ, 49010, Дніпропетровсък, E-mail:

gorbonos.so@gmail.com

Досліджено задачу оптимального керування еліптичною системою на класі необмежених мір Радона. Характерною особливістю наведеного класу задач є те, що керуваннями виступають функції з вагового простору Лебега $L^{1}(\Omega, \delta)$. Залучивши концепцію ослаблених розв'язків, отримали достатні умови розв'язності даної задачі оптимізації.

Ключові слова: задача оптимізації, простір Лоренца, ваговий простір Лебега, ослаблений розв'язок.

\section{1. Вступ}

Об’єктом досліджень даної роботи є задача оптимального керування для лінійного еліптичного рівняння з умовою Діріхле на межі області. Особливістю такого класу задач є та обставина, що керуваннями є функції з вагового простору Лебега $L^{1}(\Omega, \delta)$. За таких керувань для відповідної крайової задачі можуть не існувати слабкі розв'язки в класичних просторах Соболєва. Слід зауважити, що відповідна крайова задача не має і класичних розв'язків, оскільки коефіцієнти відповідного оператора не є диференційовними. Тому мета цієї роботи полягає в тому, щоб установити достатні умови розв'язності відповідної задачі оптимального керування, залучаючи концепцію ослаблених розв'язків, яку нещодавно було наведено у працях Ж. М. Ракотосона.

\section{2. Попередні результати та позначення}

Наведемо основні позначення, поняття та факти функціонального аналізу, необхідні для подальшого розгляду задачі.

Нехай $\Omega$ - відкрита, обмежена множина з гладкою межею простору $\mathbb{R}^{N}$, де $N \geq 2$. Через $|E|$ будемо позначати міру Лебега будь-якої вимірної множини $E \subset \mathbb{R}^{N}$, а $\chi_{E}-$ характеристична функція множини $E \subset \Omega$. Надалі покладемо: $\partial_{i}=\frac{\partial}{\partial x_{i}}, \partial_{i j}=\frac{\partial}{\partial x_{i} \partial x_{j}}$. Введемо до розгляду лінійний оператор $L$ :

$$
L u=-\sum_{i, j=1}^{N} \partial_{i}\left(a_{i j}(x) \partial_{j} u\right)+\sum_{i=1}^{N} b^{i}(x) \partial_{i} u+c_{0}(x) u,
$$

(c) С. О. Горбонос, 2012 
де $a_{i j} \in C^{0,1}(\bar{\Omega}), b^{i} \in C^{0,1}(\bar{\Omega}), c_{0} \in L^{\infty}(\Omega)$. Для будь-яких $\xi=\left(\xi_{1}, \ldots, \xi_{N}\right) \in$ $\mathbb{R}^{N}$ і для деяких $\alpha>0$ мають місце такі оцінки:

$$
\sum_{i, j} a_{i j}(x) \xi_{i} \xi_{j} \geq \alpha|\xi|^{2}, \quad c_{0}(x)-\frac{1}{2} \sum_{i=1}^{N} \partial_{i} b^{i}(x) \geq 0 \quad \text { м.с. на } \Omega \text {. }
$$

Тоді спряженим оператором до $L$ буде такий:

$$
L^{*} \varphi=-\sum_{i, j=1}^{N} \partial_{j}\left(a_{i j}(x) \partial_{i} \varphi\right)-\sum_{i=1}^{N} \partial_{i}\left(b^{i} \varphi\right)+c_{0}(x) \varphi .
$$

Нехай $u: \Omega \rightarrow \mathbb{R}$ довільна вимірна функція. Пов'яжемо з нею такі функції:

$$
\begin{gathered}
\left.u_{*}: \Omega_{*}=\right] 0,|\Omega|\left[\rightarrow \mathbb{R}, \quad u_{*}(s)=\inf \{t \in \mathbb{R}:|u>t| \leq s\},\right. \\
u_{*}(0)=\operatorname{ess} \sup _{\Omega} u, \quad u_{*}(|\Omega|)=\operatorname{essinf}_{\Omega} u, \\
u_{* *}(t)=\frac{1}{t} \int_{0}^{t} u_{*}(s) d s, \quad \text { де } t \in \Omega_{*}=(0,|\Omega|) .
\end{gathered}
$$

Слід зазначити, що функція $u_{*} \in$ спадною.

Уведемо до розгляду ваговий простір Лебега:

$$
L^{1}(\Omega, \delta)=\left\{f: \Omega \rightarrow \mathbb{R} \quad \text { вимірна за Лебегом: } \int_{\Omega}|f(x)| \delta(x) d x<+\infty\right\},
$$

де $\delta(x)=\operatorname{dist}(x, \partial \Omega)$ для будь-якого $x \in \Omega$.

Означимо простори Лоренца. У випадку коли $1<p<+\infty$ i $1 \leq q<+\infty$, покладемо:

$$
L^{p, q}(\Omega)=\left\{v: \Omega \rightarrow \mathbb{R} \text { - вимірна, } \quad|v|_{L^{p, q}}^{q}=\int_{0}^{|\Omega|}\left[t^{\frac{1}{p}}|v|_{* *}(t)\right]^{q} \frac{d t}{t}<+\infty\right\} .
$$

Для $1<p<+\infty$ i $q=+\infty$ має місце інше подання:

$$
L^{p, q}(\Omega)=\left\{v: \Omega \rightarrow \mathbb{R} \text {-вимірна, }|v|_{L^{p, \infty}}=\sup _{t \leq|\Omega|} t^{\frac{1}{p}}|v|_{* *}(t)<+\infty\right\} .
$$

Зауважимо, що $L^{p, q}(\Omega) \subset L^{p, p}(\Omega)=L^{p}(\Omega)$ для $\forall p \geq q \geq 1$. Крім того, для просторів Лоренца має місце така лема. 
Лема 2.1. [1] Нехай $L^{*}$ - вищезгаданий лінійний оператор, $L^{*}-$ спряжений до L. Тоді існуе константа $c\left(\Omega, L^{*}\right)>0$ така, що для будъ-якого $g \in L^{N, 1}(\Omega)$ існуе функція $\varphi \in W^{2}\left(\Omega,|\cdot|_{N, 1}\right) \cap H_{0}^{1}(\Omega)$, яка задовольняе наступну рівність

$$
L^{*} \varphi=g
$$

i мае місце наступна оцінка

$$
|\varphi|_{H_{1}}+\max _{i, j}\left|\partial_{i j} \varphi\right|_{L^{N, 1}} \leq c\left(\Omega, L^{*}\right)|g|_{L^{N, 1}} .
$$

Зауваження 2.1. Посилаючись на роботу [1] Ракотосона, зазначимо, що простір $L^{N, 1}(\Omega)$ є дуальним до простору $L^{N^{\prime}, \infty}(\Omega)$.

Слід зазначити, що для просторів Лоренца має місце таке подання норми:

$$
\|x\|=\int_{0}^{\infty} x^{*}(t) d \varphi(t),
$$

де $x^{*}(t)$ - невід'ємна спадна неперервна зліва функція на $(0, \infty) ; \varphi(t)$ - зростаюча увігнута функція на $[0, \infty)$ і $\varphi(0)=0$.

Зауважимо, що простори Лоренца не є рефлексивними. Разом з тим, має місце такий результат.

Лема 2.2. [3] Простір Лоренца сепарабелъний тоді і тілъки тоді, коли

$$
\varphi(+0)=0, \quad \varphi(\infty)=\infty .
$$

Наведемо далі низку результатів, які пов'язані з поняттям *-слабкої збіжності в нормованих просторах.

Означення 2.1. Нехай $Y$ - банахів простір, а $X$ - дуальний до нього простір. Тоді послідовність $\left\{u_{n}\right\}_{k=1}^{\infty} \subset X$ називають *-слабко збіжною до елемента $u \in X\left(u_{n} \stackrel{*}{\rightarrow} u\right)$ в $X$, якщо

$$
\left\langle u_{n}, u^{*}\right\rangle_{X, Y} \rightarrow\left\langle u, u^{*}\right\rangle_{X, Y} \quad \forall u^{*} \in Y .
$$

Теорема 2.1. (Теорема про напівнеперервність знизу норми відносно *-слабкої збіжності). Нехай $Y-$ нормований простір, $X=(Y)^{*} i$ нехай $\left\{x_{n}\right\}_{n \in \mathbb{N}}$ послідовність в $X$ i $x_{n} \stackrel{*}{\rightarrow} x$ в $X$ :

$$
\begin{gathered}
\left\langle x_{n}, y\right\rangle_{Y^{*}, Y} \rightarrow\langle x, y\rangle_{Y^{*}, Y} \quad \forall y \in Y, \quad \text { mo } \\
\lim _{n \rightarrow \infty} \inf \left\|x_{n}\right\|_{X} \geq\|x\|_{X} .
\end{gathered}
$$

Теорема 2.2. (Теорема Банаха - Алаоглу). Нехай $X$ - нормований простір, який е дуальним до нормованого сепарабельного простору $Y$. Нехай $K$ - обмежена, замкнена, не порожня підмножина простору $X$, тоді вона $\epsilon$ компактною відносно *-слабкої збіжнності:

$$
\forall\left\{u_{k}\right\}_{k \in \mathbb{N}} \exists\left\{u_{k_{i}}\right\}_{i \in \mathbb{N}} \subset K i \exists u^{*} \in K: \quad u_{k_{i}} \stackrel{*}{\rightarrow} u^{*} .
$$




\section{3. Поняття ослабленого розв'язку та достатні умови розв'язності задачі Діріхле}

У цьому параграфі наведемо умови, за яких є розв'язною наступна крайова задача:

$$
\begin{aligned}
& L v=f \quad \text { в } \Omega, \\
& v=0 \quad \text { на } \quad \partial \Omega .
\end{aligned}
$$

Тут $f \in L^{1}(\Omega, \delta)$, де $\delta=\operatorname{dist}(x, \partial \Omega)$ для будь-якого $x \in \Omega$.

Зауважимо, що внаслідок приналежності правої частини рівняння (3.1) до вагового простору Лебега $L^{1}(\Omega, \delta)$, вихідна задача (3.1)-(3.2) вже не може бути зведеною до класичної варіаційної постановки, оскільки в цьому випадку $f$ не є елементом простору $H^{-1}(\Omega)$, що означає: далеко не кожна функція вагового простору $L^{1}(\Omega, \delta)$ породжує лінійний неперервний функціонал на просторі Соболєва. Таким чином, залучення класичної концепції слабких розв'язків є неможливим. Зрозуміло, що задача (3.1)-(3.2) в силу зроблених припущень не має також і класичних розв'язків (недиференційовність коефіцієнтів $\left.a_{i j}\right)$. Отже, виникає нагальна потреба в ослабленні тлумачення поняття розв'язку задачі (3.1)-(3.2). У зв'язку з цим візьмемо за основу концепцію ослаблених розв'язків, яку запропоновано в праці [1].

Означення 3.1. Нехай $f \in L^{1}(\Omega, \delta)$. Тоді ослабленим розв'язком задачі (3.1)-(3.2) будемо називати функцію $v$, яка задовольняе інтегральну тотожність:

$$
\int_{\Omega} v L^{*} \varphi d x=\int_{\Omega} f \varphi d x, \quad \forall \varphi \in W^{2}\left(\Omega,|\cdot|_{N, 1}\right) \cap H_{0}^{1}(\Omega),
$$

де $W^{2}\left(\Omega,|\cdot|_{p, q}\right)=\left\{v \in W^{2,1}(\Omega): \partial_{i j} v \in L^{p, q}(\Omega)\right.$ для $\left.(i, j) \in\{1, \ldots, N\}^{2}\right\}$.

Зауважимо, що коректність такого означення розв'язку задачі (3.1)-(3.2) має сенс у силу леми 2.1 , зауваження 2.1 та наступного результату.

Теорема 3.1. Нехай $f \in L^{1}(\Omega, \delta) i N^{\prime}=\frac{N}{N-1}$. Тоді існуе єдина функція $v \in L^{N^{\prime}}, \infty(\Omega)$, яка задовольняе інтегральну тотожність

$$
\int_{\Omega} v L^{*} \varphi d x=\int_{\Omega} f \varphi d x, \quad \forall \varphi \in W^{2}\left(\Omega,|\cdot|_{N, 1}\right) \cap H_{0}^{1}(\Omega) .
$$

Крім того існуе константа $c(\Omega, L)>0$ така, що мае місце апріорна оцінка для ослабленого розв'язку $v$

$$
|v|_{L^{N^{\prime}, \infty}} \leq c(\Omega, L)|f|_{L^{1}(\Omega, \delta)} .
$$




\section{4. Про оптимізацію еліптичних систем на класі необмежених мір Радона}

Нехай об’єктом керування виступає така крайова задача:

$$
\begin{gathered}
L y=f+u \quad \text { на } \Omega \\
y=0 \quad \text { на } \quad \partial \Omega,
\end{gathered}
$$

де $f \in L^{1}(\Omega, \delta), \delta=\operatorname{dist}(x, \partial \Omega)$. Будемо казати, що $u \in$ допустимим керуванням, якщо

$$
u \in \mathbb{U}
$$

де $\mathbb{U}$ - деяка наперед задана множина простору $L^{1}(\Omega, \delta)$.

Уведемо до розгляду поняття множини допустимих розв'язків:

$$
\begin{gathered}
\Xi=\left\{(u, y) \in L^{1}(\Omega, \delta) \times L^{N^{\prime}, \infty}(\Omega):\right. \\
\left.\forall \varphi \in H_{0}^{1}(\Omega) \cap W^{2}\left(\Omega,|\cdot|_{N, 1}\right) \text { має місце } \int_{\Omega} y L^{*} \varphi d x=\int_{\Omega}(f+u) \varphi d x\right\} .
\end{gathered}
$$

Задача оптимального керування полягає в тому, щоб знайти таку пару $\left(u^{0}, y^{0}\right) \in \Xi$, на якій функціонал вартості

$$
I(u, y)=\left\|y-y^{*}\right\|_{L^{N^{\prime}, \infty}(\Omega)}+\|u\|_{L^{1}(\Omega, \delta)} \rightarrow \inf
$$

досягає свого найменшого можливого значення.

Як приклад множини допустимих керувань у задачі (4.1)-(4.4) можна взяти таку множину:

$$
\mathbb{U}_{\delta}=\left\{u(\cdot) \in L^{1}(\Omega, \delta) \quad \text { така, що }\|u\|_{B V(\Omega)} \leq C\right\} .
$$

Справді, нехай $u \in \mathbb{U}_{\delta}$, де $\mathbb{U}_{\delta} \subset L^{1}(\Omega, \delta)$, тоді для $\forall u \in \mathbb{U}_{\delta}$ має місце

$$
u(x)=\delta^{-1}(x) g(x), \quad \text { де } g \in L^{1}(\Omega) .
$$

Тоді, якщо $\mathbb{U} \subset L^{1}(\Omega)$, то $\mathbb{U}_{\delta}=\delta^{-1} \mathbb{U}$. Відомо, якщо множина обмежена по $B V$-нормі, то вона компактна в $L^{1}(\Omega)$. Отже, нехай $\mathbb{U}$ обмежена по $B V$-нормі, тоді з будь-якої послідовності $\left\{g_{n}\right\}_{n \in \mathbb{N}} \subset \mathbb{U}$ можна вилучити підпослідовність $\left\{g_{n_{k}}\right\}_{k \in \mathbb{N}}$ таку, що

$$
g_{n_{k}} \rightarrow g_{0} \in \mathbb{U} .
$$

Звідки $\delta_{k}^{-1} g_{n_{k}} \rightarrow \delta^{-1} g_{0}$. Далі нехай $u_{n_{k}}=\delta_{k}^{-1} g_{n_{k}}$ i $u_{0}=\delta^{-1} g_{0}$, тоді отримаємо:

$$
u_{n_{k}} \rightarrow u_{0} \in \mathbb{U}_{\delta} .
$$

Звідки випливає, що множина $\mathbb{U}_{\delta}$ компактна.

Наведемо основний результат даної роботи. 
Теорема 4.1. Нехай для задачі (4.1)-(4.2) мають місце такі припущення:

(i) $y^{*}$ е заданим елементом простору $L^{N^{\prime}, \infty}(\Omega)$;

(ii) множина $\mathbb{U}$ компактна відносно норми в $L^{1}(\Omega, \delta)$.

Тоді множина оптимальних розв'язків задачі (4.1)-(4.4) не е порожнъою.

Доведення. Виходячи з результатів роботи Ракотосона [1] та залучаючи теорему 3.1 , можна зробити висновок, що для довільного $u \in \mathbb{U}$ при фіксованому $f$ існує єдина функція $y=y(u) \in L^{N^{\prime}, \infty}(\Omega)$ така, що $(u, y(u)) \in \Xi$. Отже, множина $\Xi$ не є порожньою.

Таким чином, задачу (4.1)-(4.4) можна тлумачити як задачу пошуку такого керування $u$, при якому ослабленим розв'язком задачі $(4.1)-(4.2)$ був би елемент, найближчий до елемента $y^{*}$ за нормою простору $L^{N^{\prime}, \infty}(\Omega)$. Інакше кажучи, пару $\left(u^{0}, y^{0}\right) \in \Xi$ називатимемо оптимальною парою задачі (4.1)(4.4), якщо виконується умова $I\left(u^{0}, y^{0}\right)=\inf _{(u, y) \in \Xi} I(u, y)$.

Перше питання, яке виникає, пов'язане з існуванням оптимальної пари $\left(u^{0}, y^{0}\right)$. За побудовою маємо:

$$
I(u, y) \geq 0, \quad \forall(u, y) \in \Xi,
$$

звідки випливає, що $I(u, y)$ обмежений знизу. Це означає, що існує послідовність $\left\{\left(u_{k}, y_{k}\right)\right\} \in \Xi$ така, що

$$
\lim _{k \rightarrow \infty} I\left(u_{k}, y_{k}\right)=\inf _{(u, y) \in \Xi} I(u, y)
$$

Надалі називатимемо її мінімізаційною послідовністю. Далі покажемо, що 3 елементів цієї послідовності можна вилучити підпослідовність, яка буде збігатися до оптимальної пари задачі (4.1)-(4.4). 3 (4.5) маємо: $\lim _{k \rightarrow \infty} I\left(u_{k}, y_{k}\right)<$ $+\infty$. Звідки випливає, що

$$
\lim _{k \rightarrow \infty}\left\|u_{k}\right\|_{L^{1}(\Omega, \partial)}<+\infty .
$$

Це говорить про обмеженість послідовності $\left\{u_{k}\right\}$ в просторі $L^{1}(\Omega, \partial)$, проте це не $є$ достатньою умовою для висновку, що послідовність $\left\{u_{k}\right\}$ містить у собі збіжну підпослідовність. Справді, простір $L^{1}(\Omega, \delta)$ не $є$ рефлексивним, отже, не можна застосувати теорему Банаха про слабку компактність обмежених множин. Але з компактності $\mathbb{U}$ випливає, що

$$
\begin{gathered}
\exists\left\{u_{k_{l}}\right\}_{l \in \mathbb{N}} \subset\left\{u_{k}\right\}_{k \in \mathbb{N}} \quad \text { i } \quad u^{0} \in L^{1}(\Omega, \delta): \\
u_{k_{l}} \rightarrow u^{0} \quad \text { в } \quad L^{1}(\Omega, \delta),
\end{gathered}
$$

що означає

$$
\lim _{l \rightarrow \infty} \int_{\Omega}\left|u_{k_{l}}-u^{0}\right| \delta(x) d x=0 .
$$


Згідно з теоремою 3.1, для ослаблених розв'язків має місце така апріорна оцінка:

$$
\left\|y_{k}\right\|_{L^{N^{\prime}, \infty}(\Omega)} \leq C\left\|u_{k}+f\right\|_{L^{1}(\Omega, \delta)} \leq C\left(\left\|u_{k}\right\|_{L^{1}(\Omega, \delta)}+\|f\|_{L^{1}(\Omega, \delta)}\right) .
$$

Оскільки $u_{k_{l}} \rightarrow u^{0}$, то $u_{k_{l}}$ обмежена в просторі $L^{1}(\Omega, \delta)$. Отже, з (4.6) випливає, що

$$
\sup _{l \in \mathbb{N}}\left\|y_{k_{l}}\right\|_{L^{N^{\prime}, \infty}}<+\infty .
$$

Зауважимо, що простір $L^{N, 1}(\Omega)$ є дуальним до простору $L^{N^{\prime}, \infty}(\Omega)$. При цьому нормою в ньому буде

$$
\|\cdot\|_{L^{N, 1}(\Omega)}=\int_{0}^{|\Omega|} t^{\frac{1}{N}}|\cdot|_{* *}(t) \frac{d t}{t} .
$$

Нехай

$$
\begin{gathered}
\varphi^{\prime}(t)=t^{\frac{1-p}{p}} \text { або } \varphi(t)=p t^{\frac{1}{p}} \\
x^{*}(t)=|\cdot|_{* *}(t) .
\end{gathered}
$$

Тоді, врахувавши властивості функції $|\cdot|_{* *}(t)$ і залучивши лему 2.2 , отримаємо, що простір $L^{N, 1}(\Omega)$ сепарабельний.

Оскільки послідовність $y_{k_{l}}$ обмежена в $L^{N^{\prime}, \infty}(\Omega)$ і простір $L^{N, 1}(\Omega)$ є сепарабельним і дуальним до простору $L^{N^{\prime}, \infty}(\Omega)$, то за теоремою Банаха - Алаоглу знайдеться підпослідовність $\left\{y_{k_{l_{j}}}\right\}_{j \in \mathbb{N}}$ послідовності $\left\{y_{k_{l}}\right\}_{l \in \mathbb{N}}$ та елемент $y^{0} \in L^{N^{\prime}, \infty}(\Omega)$ такі, що

$$
y_{k_{l_{j}}} \stackrel{*}{\rightarrow} y^{0} \quad \text { в } \quad L^{N^{\prime}, \infty}(\Omega) \quad \forall k \in \mathbb{N} .
$$

Залучаючи попередні позначення, можемо вважати, що мінімізаційна послідовність $\left\{\left(u_{n}, y_{n}\right) \in \Xi\right\}$ є такою, що

$$
\begin{array}{lcc}
y_{n} \stackrel{*}{\rightarrow} y^{0} \quad \text { в } & L^{N^{\prime}, \infty}(\Omega) \\
u_{n} \rightarrow u^{0} & \text { в } & L^{1}(\Omega, \delta) .
\end{array}
$$

Таким чином, для мінімізаційної послідовності встановлено існування граничної пари $\left(u^{0}, y^{0}\right)$.

Тепер покажемо, що $\left(u^{0}, y^{0}\right) \in \Xi$. Очевидно, що $u^{0} \in \mathbb{U}$. Таким чином, залишається встановити, що $y^{0}=y^{0}(u)$ - ослаблений розв'язок задачі (4.1)(4.2). Оскільки $\left(u_{n}, y_{n}\right) \in \Xi$, то має місце тотожність

$$
\int_{\Omega} y_{n} L^{*} \varphi d x=\int_{\Omega}\left(f+u_{n}\right) \varphi d x .
$$


Урахувавши отримані результати в роботі Ракотосона [1] щодо граничного переходу і перейшовши до границі в (4.9), отримаємо:

$$
\int_{\Omega} y^{0} L^{*} \varphi d x=\int_{\Omega}\left(f+u^{0}\right) \varphi d x .
$$

Звідси випливає, що $y^{0}=y^{0}(u)-$ ослаблений розв'язок задачі (4.1)-(4.2), а отже $\left(u^{0}, y^{0}\right) \in \Xi$.

Залишилось показати, що $\left(u^{0}, y^{0}\right)$ - оптимальна пара. Для цього розглянемо співвідношення:

$$
\lim _{n \rightarrow \infty} I\left(u_{k}, y_{k}\right)=\inf _{(u, y) \in \Xi} I(u, y)=\lim _{n \rightarrow \infty}\left\|y_{n}-y^{*}\right\|_{L^{N^{\prime}, \infty}(\Omega)}+\lim _{n \rightarrow \infty}\left\|u_{n}\right\|_{L^{1}(\Omega, \delta)}
$$

Урахувавши (4.7)-(4.8) і скориставшись теоремою 2.1, отримаємо:

$$
\begin{gathered}
\lim _{n \rightarrow \infty}\left\|y_{n}-y^{*}\right\|_{L^{N^{\prime}, \infty}(\Omega)} \geq\left\|y^{0}-y^{*}\right\|_{L^{N^{\prime}, \infty}(\Omega)}, \\
\lim _{n \rightarrow \infty}\left\|u_{n}\right\|_{L^{1}(\Omega, \delta)}=\left\|u^{0}\right\|_{L^{1}(\Omega, \delta)} .
\end{gathered}
$$

Використавши (4.10)-(4.11), маємо:

$$
\underline{\varliminf_{n \rightarrow \infty}} I\left(u_{k}, y_{k}\right)=\inf _{(u, y) \in \Xi} I(u, y) \geq\left\|y^{0}-y^{*}\right\|_{L^{N^{\prime}, \infty}(\Omega)}+\left\|u^{0}\right\|_{L^{1}(\Omega, \delta)} .
$$

3 іншого боку,

$$
\lim _{n \rightarrow \infty} \inf _{(u, y) \in \Xi} I\left(u_{k}, y_{k}\right)=\inf _{(u, y) \in \Xi} I\left(u_{0}, y_{0}\right) .
$$

Звідки випливає, що

$$
I\left(u^{0}, y^{0}\right)=\inf _{(u, y) \in \Xi} I(u, y) .
$$

Таким чином, теорему доведено. (4.4).

Далі наведемо результат, який торкається єдиності розв'язку задачі (4.1)-

Теорема 4.2. Нехай для задачі (4.1)-(4.4) виконуються умови теореми 4.1. Якщо множина $\mathbb{U}$ опукла, то оптимальна пара для задачі (4.1)-(4.4) едина.

Доведення. Припустимо протилежне, тобто нехай існує дві оптимальні пари задачі (4.1)-(4.4)

$$
\left(u_{i}^{0}, y_{i}^{0}\right) \in \Xi, \quad i=1,2
$$

такі, що

$$
I\left(u_{1}^{0}, y_{1}^{0}\right)=I\left(u_{2}^{0}, y_{2}^{0}\right)=\inf _{(u, y) \in \Xi} I(u, y)
$$

У свою чергу,

$$
\int_{\Omega} y_{1}^{0} L^{*} \varphi d x=\int_{\Omega}\left(f+u_{1}^{0}\right) \varphi d x
$$




$$
\int_{\Omega} y_{2}^{0} L^{*} \varphi d x=\int_{\Omega}\left(f+u_{2}^{0}\right) \varphi d x .
$$

Покладемо $u^{*}=\frac{u_{1}^{0}+u_{2}^{0}}{2}, \quad y^{*}=\frac{y_{1}^{0}+y_{2}^{0}}{2}$. Звідки випливає, що

$$
\left(u^{*}, y^{*}\right)=\frac{1}{2}\left(\left(u_{1}^{0}, y_{1}^{0}\right)+\left(u_{2}^{0}, y_{2}^{0}\right)\right)
$$

Тоді за нерівністю Ієнсена маємо, що $I\left(u^{*}, y^{*}\right)<\frac{1}{2}\left(I\left(u_{1}^{0}, y_{1}^{0}\right)+I\left(u_{2}^{0}, y_{2}^{0}\right)\right)$. Урахувавши (4.12), отримаємо: $I\left(u^{*}, y^{*}\right)<\inf _{(u, y) \in \Xi} I(u, y)$, але це не можлиBо.

3 іншого боку $\left(u^{*}, y^{*}\right) \in \Xi$. Справді, оскільки $\mathbb{U}$ опукла, то $u^{*} \in \mathbb{U}$. Далі помножимо (4.13) i (4.14) на $1 / 2$, а потім додамо. В результаті отримаємо:

$$
\int_{\Omega} y^{*} L^{*} \varphi d x=\int_{\Omega}\left(f+u^{*}\right) \varphi d x,
$$

звідки випливає, що $y^{*}-$ ослаблений розв'язок задачі (4.1)-(4.2). Отже, доходимо висновку: $\left(u^{*}, y^{*}\right) \in \Xi$, що і доводить хибність зроблених припущень. Теорему доведено.

\section{5. Про існування оптимальних керувань на класі ослаблених розв'язків для некоректно поставлених задач}

Тепер покажемо, що задачі оптимального керування мають суттєві відмінності від задач математичної фізики. А саме, вони можуть бути коректно поставленими (з точки зору їх розв'язності) для погано обумовлених систем. Справді, розглянемо наступну задачу:

$$
\begin{gathered}
I(u, y)=\left\|y-y^{*}\right\|_{L^{N^{\prime}, \infty}(\Omega)}+\|u\|_{L^{1}(\Omega, \delta)} \rightarrow \mathrm{inf}, \\
L y=u \text { на } \Omega, \\
u \in \mathbb{U},
\end{gathered}
$$

де $y^{*}$ - заданий елемент простору $L^{N^{\prime}, \infty}(\Omega), \mathbb{U}-$ не порожня компактна опукла підмножина простору $L^{1}(\Omega, \delta)$, а функціонал $I(u, y)$ строго опуклий.

Зауважимо, що задача (5.2) є некоректно поставленою крайовою задачею, оскільки тут відсутні будь-які крайові умови. Отже, така задача допускає існування безлічі розв'язків.

Надалі через $\Xi$ позначимо множину допустимих розв'язків задачі (5.1)(5.3):

$$
\begin{gathered}
\Xi=\left\{(u, y) \in L^{1}(\Omega, \delta) \times L^{N^{\prime}, \infty}(\Omega):\right. \\
\left.\forall \varphi \in H_{0}^{1}(\Omega) \cap W^{2}\left(\Omega,|\cdot|_{N, 1}\right) \text { має місце } \int_{\Omega} y L^{*} \varphi d x=\int_{\Omega} u \varphi d x\right\} .
\end{gathered}
$$


Таким чином, задача оптимального керування полягає в тому, щоб знайти таку пару $\left(u^{0}, y^{0}\right) \in \Xi$, на якій функціонал (5.1) досягає свого найменшого можливого значення.

Наведемо результат, який торкається існування та єдиності розв'язку задачі оптимального керування (5.1)-(5.3).

Теорема 5.1. Задача (5.1)-(5.3) мае единий розв'язок $\left(u^{0}, y^{0}\right) \in L^{1}(\Omega, \delta) \times$ $L^{N^{\prime}, \infty}(\Omega)$.

Доведення. Схема доведення теореми аналогічна доведенням теорем (4.1) і (4.2), окрім перевірки того факту, що множина $\Xi$ не $є$ порожньою, оскільки відповідна крайова задача некоректно поставлена. Отже, потрібно лише показати, що множина $\Xi$ не $є$ порожньою. Нехай $u \in \mathbb{U}$, а $y(x) \in L^{N^{\prime}, \infty}(\Omega)$. Тоді за теоремою 3.1 існує єдиний ослаблений розв'язок задачі:

$$
L y=u \quad \text { на } \Omega,\left.\quad y\right|_{\partial \Omega}=0,
$$

тобто має місце така інтегральна тотожність:

$$
\int_{\Omega} y L^{*} \varphi d x=\int_{\Omega} u \varphi d x \quad \forall \varphi \in H_{0}^{1}(\Omega) \cap W^{2}\left(\Omega,|\cdot|_{N, 1}\right) .
$$

Звідси випливає, що $(u, y) \in \Xi$, а, отже, $\Xi$ не $є$ порожньою.

\section{6. Висковки}

Залучаючи поняття ослабленого розв'язку у формі означення 3.1 , отримали достатні умови розв'язності задачі (4.1)-(4.4) на класі ослаблених розв'язків з простору Лоренца. Крім цього показано, що задача оптимального керування є розв'язною для некоректно поставленої крайової задачі (5.2).

\section{Бібліографічні посилання}

1. Rakotoson $J$. M. On the differentiability of very weak solutions with right-hand side data integrable wiht respect to the distance to the boundary/ J. M. Rakotoson, J. I. Diaz // Journal of Functional Analysis. - 2009. - no. 257. - P. 807-831.

2. Rakotoson J. M. Rearrangement Relatif: un instrument d'estimation dans les problemes aux limites / J. M. Rakotoson. - Berlin: Springer-Verlag, 2008.

3. Крейн С. Г. Интерполяция линейних операторов / С. Г. Крейн, Ю. И. Петунин, Е. М. Семенов. - М.: Наука, 1978.

4. Когут П. І. Оптимізація в нелінійних еліптичних крайових задачах / П. І. Когут, О. А. Рядно, О. П. Когут. - Дніпропетровськ : ДДФА, 2010.

5. Фурсиков А. В. Оптимальное управление распределенными системами. Теория и приложения / А. В. Фурсиков. - Новосибирск : Научная книга, 1999. 